\title{
Binaries among low-mass stars in nearby young moving groups $\star \star \star \star$
}

\author{
Markus Janson ${ }^{1}$, Stephen Durkan ${ }^{2}$, Stefan Hippler ${ }^{3}$, Xiaolin Dai $^{3}$, Wolfgang Brandner ${ }^{3}$, Joshua Schlieder ${ }^{4}$, \\ Mickaël Bonnefoy ${ }^{5}$, and Thomas Henning ${ }^{3}$ \\ 1 Department of Astronomy, Stockholm University, 11419 Stockholm, Sweden \\ e-mail: markus. janson@astro.su.se \\ 2 Astrophysics Research Center, Queens University Belfast, Belfast, BT7 1NN, UK \\ 3 Max Planck Institute for Astronomy, 69117 Heidelberg, Germany \\ 4 NASA Exoplanet Science Institute, Caltech, Pasadena, California, CA 91125, USA \\ 5 Univ. Grenoble Alpes, IPAG, 38058 Grenoble, Cedex 9, France
}

Received 23 October 2016 / Accepted 2 December 2016

\begin{abstract}
The solar galactic neighborhood contains a number of young co-moving associations of stars (known as young moving groups) with ages of $\sim 10-150 \mathrm{Myr}$, which are prime targets for a range of scientific studies, including direct imaging planet searches. The latetype stellar populations of such groups still remain in their pre-main sequence phase, and are thus well suited for purposes such as isochronal dating. Close binaries are particularly useful in this regard since they allow for a model-independent dynamical mass determination. Here we present a dedicated effort to identify new close binaries in nearby young moving groups, through highresolution imaging with the AstraLux Sur Lucky Imaging camera. We surveyed 181 targets, resulting in the detection of 61 companions or candidates, of which 38 are new discoveries. An interesting example of such a case is 2MASS J00302572-6236015 AB, which is a high-probability member of the Tucana-Horologium moving group, and has an estimated orbital period of less than $10 \mathrm{yr}$. Among the previously known objects is a serendipitous detection of the deuterium burning boundary circumbinary companion 2MASS J01033563-5515561 (AB) $b$ in the $z^{\prime}$ band, thereby extending the spectral coverage for this object down to near-visible wavelengths.
\end{abstract}

Key words. binaries: visual - stars: low-mass - stars: pre-main sequence

\section{Introduction}

Young moving groups (YMGs) are associations of stars that, in addition to having individual indications of youth, are clustered in phase space and therefore generally assumed to originate from a common birth cluster (e.g., Torres et al. 2000; Zuckerman et al. 2001). Thus, they can be expected to be approximately co-eval, which opens up a range of scientific opportunities that are otherwise unattainable. For instance, statistical age estimators can be applied to a large number of stars in a YMG in order to average out the scatter and improve the precision of the age and, conversely, if the age of individual stars can be determined with particularly good accuracy, this can in principle be applied to the age estimation of all other stars that are associated with the same YMG. The age is a fundamental parameter for many purposes in stellar science, but one of the main reasons for why it has received particular attention in recent years is its relevance for exoplanet research. Since planets are hottest directly after formation and subsequently cool gradually for the rest of their lifetimes (or until they reach a thermal equilibrium due to illumination from a parent star), the interpretation of a directly imaged planet depends crucially on understanding its age (e.g., Marois et al. 2008; Lagrange et al. 2010; Kuzuhara et al. 2013).

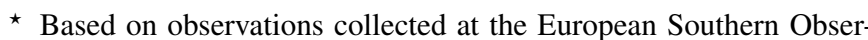
vatory, Chile (Programs 096.C-0243 and 097.C-0135).

$\star \star$ Tables $1-3$ are only available at the CDS via anonymous ftp to cdsarc.u-strasbg.fr (130.79.128.5) or via

http://cdsarc.u-strasbg.fr/viz-bin/qcat?]/A+A/599/A70
}

Since YMGs are additionally prime targets for direct imaging surveys owing to their youth and proximity (e.g., Chauvin et al. 2010; Biller et al. 2013; Brandt et al. 2014), understanding the ages of YMGs is closely coupled to the understanding of directly imaged planets.

M-type stars have rather long pre-main sequence lifetimes of 100 Myr (e.g., Baraffe et al. 1998). As a result, when such stars reside in YMGs, they are typically still evolving through the pre-main sequence phase, and thus they can potentially be isochronally dated to a good level of precision. Such isochronal dating can in principle be performed using a $T_{\text {eff }}$ versus $L_{\text {bol }}$ relationship (e.g., Janson et al. 2007), but the temperature scale of M-dwarfs is highly uncertain and thus a potential cause of systematic error. A more robust analysis can be based on using an $M_{\text {star }}$ versus $L_{\text {bol }}$ relationship instead. This has been achieved in a few cases (e.g., Bonnefoy et al. 2009; Köhler et al. 2013; Montet et al. 2015a), but more examples would be highly beneficial for covering more YMGs and more reference cases in each YMG to test for robustness, coevality within YMGs, etc. In previous high-resolution imaging surveys, we identified a large number of binaries in young systems (Bergfors et al. 2010; Janson et al. 2012a). However, not all of these can be associated with known YMGs, and only a subset of the discovered binaries have orbital periods that are short enough so that robust orbital parameters can be estimated in a reasonably rapid timeframe (Janson et al. 2014a). In order to increase the yield of high-utility binaries, it would be more efficient to target stars that have already been identified as YMG members. 
Here, we present the results of a high-resolution imaging survey of low-mass stars that have been identified as probable members of nearby YMGs (Malo et al. 2013, 2014; Kraus et al. 2014), primarily motivated by the reasoning above. In the following, we will first present the selected sample of stars in Sect. 2 and the acquisition and reduction of the imaging data in Sect. 3. We will then discuss the results for the sample at large in Sect. 4.1, pay special attention to the "planetary mass" companion 2MASS J01033563-5515561(AB)b in Sect. 4.2 and note peculiarities of other targets in Sect. 4.3. Finally, we will summarize the results of the survey in Sect. 5.

\section{Target sample}

Our targets were selected from three catalogues of late-type stars identified as high-probability members of nearby YMGs (Malo et al. 2013, 2014; Kraus et al. 2014) that had not been previously monitored with AstraLux. In principle, six observational parameters are required to exactly relate the $X Y Z U V W$ of the target to that of various moving groups it could conceivably be associated with, but since late-type stars are faint at visiblelight wavelengths, most potential YMG members lack a parallactic distance, and many lack a radial velocity measurement, so a probabilistic estimation must sometimes be made on the basis of only four parameters (right ascension, declination, and proper motion along each of these directions). Nonetheless, the Bayesian estimations made in, e.g., Malo et al. (2014) using the BANYAN code can often distinguish field objects from moving group members with this constrained parameter set with quite high probabilities, provided that the priors are trustworthy.

For the purpose of scheduling and executing the observations, we took the membership assignments in the aforementioned catalogues at face value. However, since then the first Gaia data release has been presented (Gaia Collaboration 2016), including the Tycho-Gaia Astrometric Solution (TGAS, see Michalik et al. 2015). This contains new parallaxes and improved proper motions for 29 of the observed targets. Hence, for these cases we re-evaluated YMG membership using the BANYAN II tool (Gagné et al. 2014) with the updated astrometry from Gaia. In many cases, the Gaia parallactic distance was remarkably close to the kinematic distance prediction yielded by BANYAN when no measured parallax is provided. Some particularly notable cases are mentioned in Sect. 4.3. In this way, we found that the YMG membership hypothesis is supported (and strengthened) by the addition of a Gaia parallax in 17 of the 29 cases. However, in the other 12 cases, membership could not be supported, with the YMG probability effectively going down to $0 \%$ in some cases. This relatively high rejection rate should be taken as a caution that the other targets, for which no parallactic distance exists yet, should still be taken only as candidate YMG members rather than bona fide members. Still, a majority of the re-assessed cases were verified as YMG members, which can be seen as an indication that most of the other candidate assignments are probably also correct. In due time, Gaia will provide accurate distances to all of these targets, such that membership can be more robustly assessed across the board.

The sample properties are listed in Table 1.

\section{Observations and data reduction}

The observations used in this survey were taken during three separate runs during the European Southern Observatories (ESO) observing periods P96 (program 096.C-0243) and P97 (program
097.C-0135). Two of the runs were scheduled in P96, with four nights spanning 24-27 Nov. 2015 and another four nights spanning 24-27 Dec. 2015. Unfortunately, the November run suffered from very poor conditions with clouds, strong winds, and poor seeing, so only a few targets could be usefully observed for the survey on the night of Nov. 27. The December run was considerably better and yielded useful data every night. In P97, a single four-night run was scheduled for 16-19 May 2016. One of these nights, May 17, was very productive and offered a clear sky and good seeing for most of the night, but like the Nov. run, the rest of the time was clouded out.

For all of the observations, we used the AstraLux Sur Lucky Imaging camera (Hippler et al. 2009) mounted at the guest instrument port of the $3.5 \mathrm{~m}$ New Technology Telescope (NTT) in La Silla. The $z^{\prime}$ filter was used, and typically 10000 frames with individual integration times of a few tens of ms were acquired for each target. In total, we observed 181 separate targets during the available clear nights. Nine of these were observed twice, since some of the binary candidates detected in the late 2015 runs were re-observed in the May 2016 run. Generally, a subarray readout of $256 \times 256$ pixels was used since we are primarily interested in close binaries, but in the case of wide binaries, where both components may be interesting or where it was not immediately obvious to the observer which star was the actual target, the full $512 \times 512$ detector was read out in order to maintain both components firmly within the field of view. The field of view for the full frame is approximately $16^{\prime \prime} \times 16^{\prime \prime}$.

Data reduction was done in an identical way to our previous AstraLux surveys (e.g., Janson et al. 2012a, 2014a). The reduction pipeline (Hormuth et al. 2008) produces several Lucky Imaging outputs with different levels of selection (e.g., all frames used, the $10 \%$ best seeing frames used, the $1 \%$ best seeing frames used, etc.). For our purposes, we consistently chose a $10 \%$ selection for further analysis as it provides a good tradeoff between resolution and sensitivity. The final pixel scale as well as the true north orientation were determined individually for the different runs by observing clusters that had been previously observed with the Hubble Space Telescope (HST) for astrometric reference. For the Nov. 2015 and Dec. 2015 runs, we used Trapezium for this purpose with HST coordinates from McCaughrean et al. (1994), and for the May 2016 run, we used M15 with HST coordinates from van der Marel et al. (2002). In this way, we found pixel scales of 15.19 mas/pixel for the Nov. 2015 run, 15.20 mas/pixel for the Dec. 2015 run, and 15.27 mas/pixel for the May 2016 run. Likewise, we derived true north orientations of $2.17 \mathrm{deg}$ for Nov. 2015, $2.40 \mathrm{deg}$ for Dec. 2015, and $3.04 \mathrm{deg}$ for May 2016. The calibration uncertainties are in the range $0.06-0.13 \mathrm{mas} / \mathrm{pixel}$ in pixel scale and $0.16-0.30 \mathrm{deg}$ in true north orientation.

From the output images, relative astrometry and photometry were derived for any pair of stars that could be identified in the images. This was again done as in our previous studies, with aperture photometry and Gaussian centroiding in the case of wide separation pairs, and point spread function (PSF) fitting for close separation pairs, where the PSFs of single stars were used as references. Triple systems consisting of a close pair and a third wide component need special attention in this regard. We handled these by choosing an aperture large enough to encompass both components of the close pair, thus acquiring an $\mathrm{AB}-\mathrm{C}$ (or A-BC) relative photometry. The relative photometry of the close pair was then determined through PSF fitting as usual, and from this the relative photometry between the brighter component of the pair and the wider component could be derived. Pairs of stars of near-equal brightness are subject to the "false triple" 


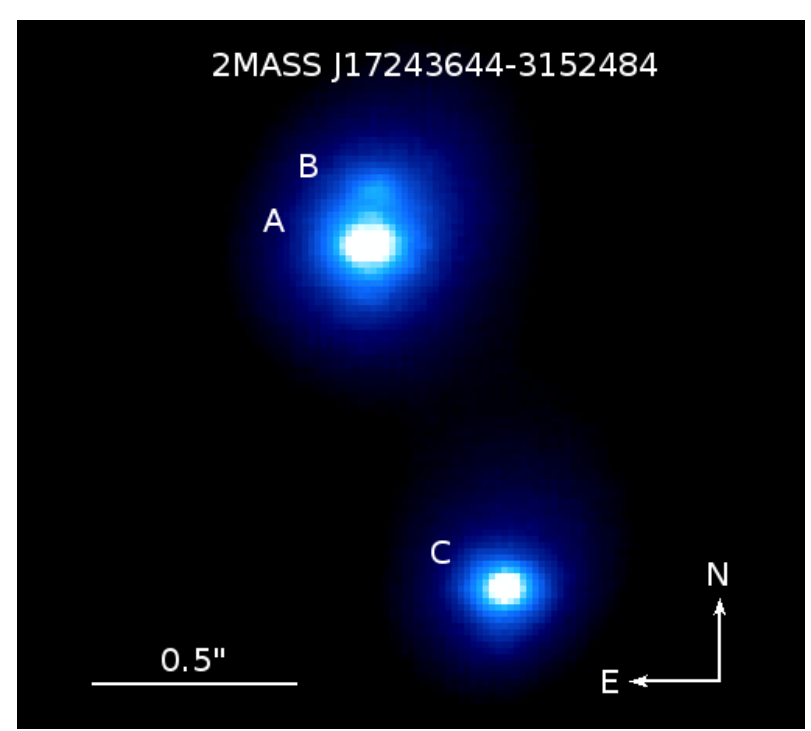

Fig. 1. J17243644-3152484 triple system, resolved by AstraLux for the first time. The YMG classification of this system is unclear (see Sect. 4.3)

effect (Law 2006), in which the pair of components map onto three apparent PSFs in the final image, and require special attention. In the context of astrometry, there is a $180 \mathrm{deg}$ ambiguity in the position angle of such cases, since it cannot be uniquely determined which of the two binary components is which (this is an issue for components of nearly equal brightness even in the absence of a false triple effect). In the context of photometry, we have shown in previous papers (Janson et al. 2012a, 2014b) that the photometric determinations on false triple pairs are biased, hence for this study, we simply omit a relative photometric analysis of such systems.

\section{Results and discussion}

\subsection{Multiplicity in the sample}

In our sample of 181 targets, we detect 61 candidate companions, 23 of which were previously reported in the Washington Double Star catalogue (Mason et al. 2001) and other sources cited individually in this article. The other 38 are, to our knowledge, new detections. An example of the new detections, the triple system J17243644-3152484, is shown in Fig. 1.

The astrometric properties of the multiple systems are summarized in Table 2. Each of the 23 companions that have been previously observed could be confirmed to share a common proper motion (CPM) with the primary. This is consistent with our experience from previous surveys (e.g., Janson et al. 2012a) that the contamination frequency is low for typical targets (i.e., targets that are not very close to the galactic plane). While we do have second epoch observations for nine companions, eight of which are new, the proper motions for several of them are too small to be statistically significantly measurable over a $\sim 5$ month baseline. J12092998-7505400 AB could nonetheless be confirmed as sharing a CPM with $>10 \sigma$ confidence. While not yet formally noted as CPM in the table, J07343426-2401353 AB and J07523324-6436308 BC could be seen as marginally confirmed CPM at $3-4 \sigma$. For the targets that do not have literature epochs and could not be confirmed as CPM, we made an additional test by checking digital sky surveys for historical epochs for the targets. This is similar to the checks performed in Janson et al. (2014b) and in other recent surveys (e.g.,
Montet et al. 2015b; Schlieder et al. 2016). The best source for such checks is typically the Palomar Observatory Sky Survey (POSS), which includes images covering a large fraction of the sky from epochs around 1950, thus providing a sufficient baseline for targets with proper motions of $\sim 100 \mathrm{mas} / \mathrm{yr}$ to move by several arcseconds on the sky, which is usually more than the seeing-limited resolution of the images. Thus, background contaminants in the AstraLux image may be separated from the primary in such archival images for sufficiently fast-moving targets. Unfortunately, most of our targets are too far south to be included in POSS. In these cases, the best option is typically the SERC survey from the UK Schmidt telescope, which has first epoch data from the late 1970s or early 1980s. Furthermore, some of our targets with candidate companions have a quite slow proper motion, down to $11 \mathrm{mas} / \mathrm{yr}$ in the slowest case. In order to be able to draw confident conclusions, we set the threshold that $3^{\prime \prime}$ of motion must have occurred between the archival epoch and the AstraLux data for the archival epoch to be useful. This leaves 15 targets that can be usefully checked. Of these, there are 10 cases in which no candidate background star can be seen in the archival image. This strongly implies that the respective candidates are physical companions. In this context it should still be considered that the filters of the two epochs are quite different; the archival epochs are at shorter wavelengths than the AstraLux data. Hence, a hypothetical very red background source may escape detection in the archival data, in which case it would be erroneous to label the candidate as CPM. We consider that a positive detection is needed for a final confirmation of CPM, and thus we label these candidates "implied CPM" rather than fully confirmed CPM. This is marked with the label "I" in Table 2. In the remaining five cases, the separation between the primary and candidate was large enough that that they could be recognized as partially resolved in the archival images, such that a real CPM test could be performed. In this way, we could confirm three additional CPM companions (J003025726236015 C, J18450097-1409053 B, and J23204705-6723209 B) and two background contaminants (J00514081-5913320 B and J23332198-1240072 B). The relatively large rejection fraction is due to the fact that these are the widest separations among the candidates, which is where the contaminants are expected to reside. Of the candidates that remain unverified, we expect that three (J05111098-4903597 B and J16572029-5343316 B and C) are possible/probable background contaminants, but the rest are very close and/or very bright relative to the primary, hence they are all probable companions. Obviously, common proper motion tests over a longer timescale will be required to verify this beyond a reasonable doubt in the individual cases.

Photometry and derived quantities are shown in Table 3. The relative photometry was used to estimate individual masses using theoretical mass-luminosity relationships for young stars. Each target system was assigned an age based on their YMG membership assignment as shown in Table 1. The ages for the AB Dor (ABMG, 150 Myr), $\beta$ Pic (bPMG, 25 Myr), Carina (CAR, 45 Myr), Columba (COL, 40 Myr), TucanaHorologium (THA, 45 Myr), and TW Hya (TWA, 10 Myr) YMGs were adopted from Bell et al. (2015). Argus is considered a questionable association by Bell et al. (2015), but is used in the BANYAN framework, so we assign it an age of 40 Myr following Torres et al. (2008). For a given star with a given age, we adopt the evolutionary models of Baraffe et al. (2015) to make predictions of the masses from the measured brightnesses. The combination of masses for the two components of a binary pair that minimizes the RMS residuals of the $\Delta z^{\prime}$ from our data and the total $J$-band magnitude from 2MASS (Skrutskie et al. 2006) 


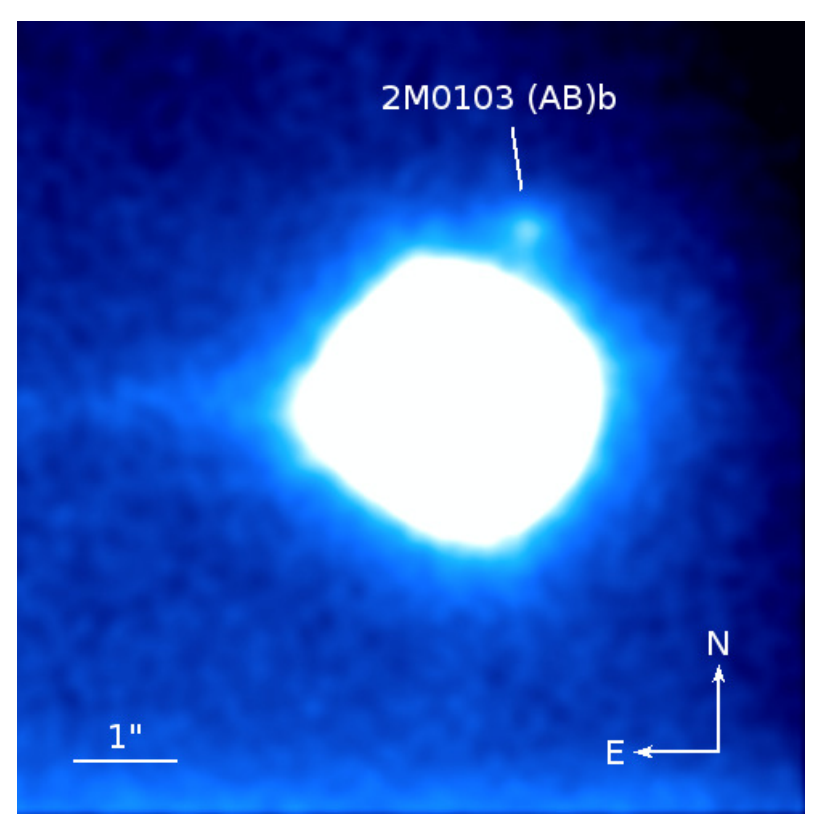

Fig. 2. AstraLux $z^{\prime}$ image of the $2 \mathrm{M} 0103$ system. Smoothing with a Gaussian kernel has been applied to better show the faint very low-mass companion first reported in Delorme et al. (2013).

is adopted for this purpose. The total mass $m_{\mathrm{tot}}$ of the binary in combination with an estimated semi-major axis $a$ can then in turn be used to make a tentative prediction for the orbital period $P$ of the pair, since $P \sim m_{\mathrm{tot}}^{-1 / 2} a^{3 / 2}$. An estimation for the semi-major axis can be acquired simply by considering that for sensible eccentricity distributions, the ratio between average projected separation and semi-major axis of a binary population is close to unity (Brandeker et al. 2006; Bonavita et al. 2016). In other words, the expectated value for the semi-major axis of a binary with no additional information about its orbit is approximately equal to its instantaneous projected separation. The period estimations resulting from this analysis are shown in Table 3. These estimations are subject to large uncertainties, for a range of reasons such as systematic uncertainties in the models and ages, as well as the broad scatter in the translation between projected separation and semi-major axis. However, prior to measuring the actual orbits this is the best that can be done with the data at hand, so it is a useful procedure for determining which systems are the most promising to follow up with a relatively high cadence for an orbital determination within a realistic timescale. Indeed, identifying such systems is the primary purpose of this study.

As mentioned previously, the false triple binaries are unsuitable for relative photometry and are thus not included in Table 3, but acquiring approximate period estimations is still of interest. For this purpose, we apply a simplified procedure of assuming that the components are approximately of equal brightness, and estimate the mass based on total $J$-band magnitude alone. Through this procedure, we acquire estimations of $8 \mathrm{yr}$ for J00302572-6236015 AB, $43 \mathrm{yr}$ for J01033563$5515561 \mathrm{AB}, 9 \mathrm{yr}$ for J02303239-4342232 AB, $295 \mathrm{yr}$ for J04475779-5035200 AB, 111 yr for J12092998-7505400 AB, $89 \mathrm{yr}$ for J17130733-8552105 AB, 39 yr for J20223306$2927499 \mathrm{AB}$, and $91 \mathrm{yr}$ for J21342935-1840372 AB. In total among the pairs studied in this survey, this means that nine pairs have an estimated orbital period $<40 \mathrm{yr}$, which we deem to be the relevant cutoff timescale for sufficiently rapid orbits to motivate regular astrometric monitoring. We particularly note the two pairs J00302572-6236015 AB and J02303239-4342232 AB which both have very rapid estimated orbital timescales of $<10$ yr. J00302572-6236015 is identified as a THA member in Kraus et al. (2014), and a BANYAN II check supports this at a very high level of probability $(99.7 \%$ THA member with standard priors), though a parallactic distance is still required for it to be formally regarded as a bona fide member. J023032394342232 has been extensively studied in a YMG context (e.g., Torres et al. 2008; Schlieder et al. 2010); in this study our baseline YMG assumption was based on the COL classification in Malo et al. (2014). Since then, a parallax has become available in TGAS which we could use to verify this membership in a BANYAN II analysis. We find that with standard priors, the probability of COL membership is $82.6 \%$, with a small probability of $15.2 \%$ that it is a member of THA instead. The probability of it being a field contaminant is only $1.9 \%$. In other words, it is very likely to be a genuine YMG member. We therefore consider J00302572-6236015 AB and J02303239-4342232 AB to be the very top priorities for astrometric follow-up out of the 61 examined in this work.

\subsection{The case of $2 \mathrm{M} 0103$}

An interesting ancillary outcome of our survey was the re-detection of J01033563-5515561(AB)b (system identifier hereafter abbreviated as $2 \mathrm{M} 0103$ ) originally reported in Delorme et al. (2013). Our image, which is displayed with a high stretch and has been smoothed with a Gaussian kernel of 20 pixel (304 mas) FWHM, can be seen in Fig. 2. 2M0103(AB)b was estimated in Delorme et al. (2013) to be a $\sim 12-14 M_{\text {jup }}$ object at a relatively wide orbit ( $\sim 84 \mathrm{AU}$ projected separation) around a close low-mass stellar binary pair. It can therefore be regarded as a possible circumbinary planet, although it seems unlikely that it could have formed through standard planet formation mechanisms, at least at its present location. The targets of our survey were selected solely on the basis of probable late-type YMG members that had not previously been observed by AstraLux, hence the inclusion of this target in the survey was entirely coincidental. Only after we had discovered the point source in the AstraLux images, and a detailed literature search was made for 2M0103, was the history of the system realized.

Special considerations are required to constrain the properties of 2M0103(AB)b because the conditions were not optimal during the observation and the companion is faint and embedded in the PSF halo of the central binary, and also because the central binary exhibits a false triple effect, further complicating matters. We estimate relative photometry by using 15 pixel circular apertures around the primary pair and around the faint companion. The contribution of the PSF halo of the primary pair was estimated by taking the mean of the aperture flux at four different locations at the same separation from the pair as the companion. The points chosen for this purpose were due north, south, and west from the primary pair, and the point directly opposite to the companion's location. The eastward direction was omitted for this purpose since the PSF halo has a coma-like extension in that direction. In this way, we found that the AB-b contrast is $\Delta z^{\prime}=6.5 \pm 0.3 \mathrm{mag}$. This is the only photometric quantity that is directly measurable in the data because of the false triple effect of the primary, but if we assume that the A and B components have approximately equal brightness, which holds true at longer wavelengths (Delorme et al. 2013), then the A-b contrast can be expected to be $\sim 5.7 \mathrm{mag}$.

For relative astrometry, we simply estimate the locations of the companion and the primary pair barycenter by eye, 
adopting a \pm 1 pixel uncertainty. This gives a separation of $1.767 \pm 0.014$ arcsec and a position angle of $335.9 \pm 0.5 \mathrm{deg}$. As can be seen in Fig. 3, the position in our Dec. 2015 image is consistent with that in the Nov. 2012 image of Delorme et al. (2013), and also confirms that orbital motion has occurred since their Oct. 2002 epoch. Given the long baseline and relatively high proper motion of 2M0103, CPM is thoroughly obvious.

While a detailed analysis of the physical properties of $2 \mathrm{M} 0103(\mathrm{AB}) \mathrm{b}$ is beyond the scope of this paper, it is interesting to assess whether the observational properties that we derive are consistent with the conclusions about the object properties in Delorme et al. (2013). In particular, we ask whether our photometry is consistent with the $\sim 12-14 M_{\text {jup }}$ mass derived there. To test this, we first take the system age of $30 \mathrm{Myr}$ adopted by Delorme et al. (2013) at face value, and use the BT-SETTL models (Baraffe et al. 2015) for an isochronal mass-brightness conversion. Given a primary mass of $\sim 0.2 M_{\text {sun }}$, the models pre$\operatorname{dict} M_{z, \mathrm{~A}}=8.3 \mathrm{mag}$, and with an A-b contrast of $5.7 \mathrm{mag}$, this gives $M_{z, \mathrm{~b}}=14.0 \mathrm{mag}$, which in turn gives a mass prediction of 13-14 $M_{\text {jup }}$ from linear interpolation of public BT-SETTL grids. This is fully consistent with the previous value derived for the system. Hence, the data give a consistent picture of a companion spectral energy distribution (SED) for a $\sim 2000 \mathrm{~K}$ object, which at an age of $30 \mathrm{Myr}$ corresponds to a mass in the range of the deuterium burning limit. However, a special note needs to be made about the age. The age assignment is based on THA membership in Delorme et al. (2013), so as a first check it is useful to test whether we can support this conclusion. Our input sample classification for 2M0103 is THA, based on Kraus et al. (2014). We have re-assessed this membership using the BANYAN II tool, which supports this classification at a very high probability of $99.95 \%$, so the membership indeed seems to be very robust. A remaining issue, then, is how old THA actually is. The $30 \mathrm{Myr}$ adopted in Delorme et al. (2013) comes from Torres et al. (2008), but in this paper we use the Bell et al. (2015) ages, which for THA gives a somewhat older age range of $\sim 40-50 \mathrm{Myr}$. This corresponds to a BT-SETTL predicted mass range of $\sim 15-20 M_{\text {jup }}$. Thus, we conclude that our data imply an SED consistent with the longer wavelength data of Delorme et al. (2013), and that our analysis supports the classification of the system as a THA member, but we note that the uncertainty on the specific age of THA broadens our error bars in mass relative to the discovery paper.

It is quite rare for a planet-like object to be detected at such a short wavelength range as in $z^{\prime}$ band. Fomalhaut $b$ has been detected at even shorter wavelengths (Kalas et al. 2008), but its observed flux does not arise from a photosphere (e.g., Janson et al. 2012b). H $\alpha$ emission related to accreting planets may have been observed in the LkCa 15 system (Sallum et al. 2015), but it remains unclear whether the planets themselves have been imaged (Kraus \& Ireland 2012; Thalmann et al. 2016). It is all the more unusual considering the rather modest size of the $3.5 \mathrm{~m}$ NTT. While the use of Lucky Imaging with AstraLux was very useful in recovering this object, its detection is arguably less a question of instrumental capabilities than a question of target properties. It is the rare combination of proximity and youth that causes an object with such a low mass like $2 \mathrm{M} 0103(\mathrm{AB}) \mathrm{b}$ to be hot and luminous enough that it emits a non-negligible amount of radiation shortward of $1 \mu \mathrm{m}$. This emphasizes that studies of YMGs performed with even modestly resource demanding instruments such as AstraLux have the potential to yield very low-mass discoveries. In this context, it also underlines the importance of following up the candidate companions yielded by the survey over longer timescales to test for CPM. While the faintest candidates in our survey are by far the most likely to be background contaminants, they are also potentially the most interesting individual objects provided by the survey.

\subsection{Individual target notes}

In this section, we make brief notes about a few targets that deserve or require particular attention.

J02303239-4342232: this system was already discussed in Sect. 4.1 because of the short estimated orbit of the AB pair. Here we additionally point out that there is a wide common proper motion companion at a separation of $173^{\prime \prime}$ (Alonso-Floriano et al. 2015). Hence, the system is at least triple.

J02442137+1057411: the YMG membership of this star was classified as ambiguous in Malo et al. (2013), but we observe it as being multiple, so a more specific membership classification would be preferable for the mass estimation of the binary components. Hence, we used the BANYAN II tool to see if some clearer YMG preference could be derived. In this analysis, $\mathrm{J} 02442137+1057411$ was assigned a $93.4 \%$ probability of being a member of the $\beta$ Pic moving group with the standard prior. We thus consider it to be a bPMG member here.

J05015881+0958587: in addition to the wide component seen in the AstraLux images, which was originally reported in Henry et al. (1997) and confirmed in Delfosse et al. (1999), the primary in the system also has a spectroscopic binary companion (Delfosse et al. 1999). With a period of only 12 days, the spectroscopic pair is, however, much too close to be resolved in our images.

J05064991-2135091: two of the three components of this system have individual 2MASS identifiers: J05064991-2135091 and J05064946-2135038. The latter has a close companion, which is the third component of the system. We used the full AstraLux frame instead of subarray readout for this target so that both wide components could be included.

J06153953-8433115: YMG membership of J061539538433115 is labeled "ambiguous" in Malo et al. (2013), but since we resolve it as a binary, it is desirable to attain a more detailed view of YMG membership for age and mass assignment purposes. This turns out to be a complicated issue for this target because BANYAN II gives only a $38.8 \%$ probability of bPMG membership versus $59.9 \%$ for the field with the standard priors. However, if we chose the priors to constrain the age to be $<1 \mathrm{Gyr}$, the probability for bPMG membership goes up to $65.9 \%$. This choice is arguably appropriate since the star is part of the Riaz et al. (2006) sample, which is indeed expected to be $<1$ Gyr. Hence, we adopt the bPMG age for this target, although we note that the evidence for such a membership is weak relative to most other targets in the survey.

J11211723-3446454: the two components of this system have individual 2MASS identifiers, J11211723-3446454 and J11211745-3446497. The full-frame field of view of AstraLux Sur was used to fit the two components simultaneously. 

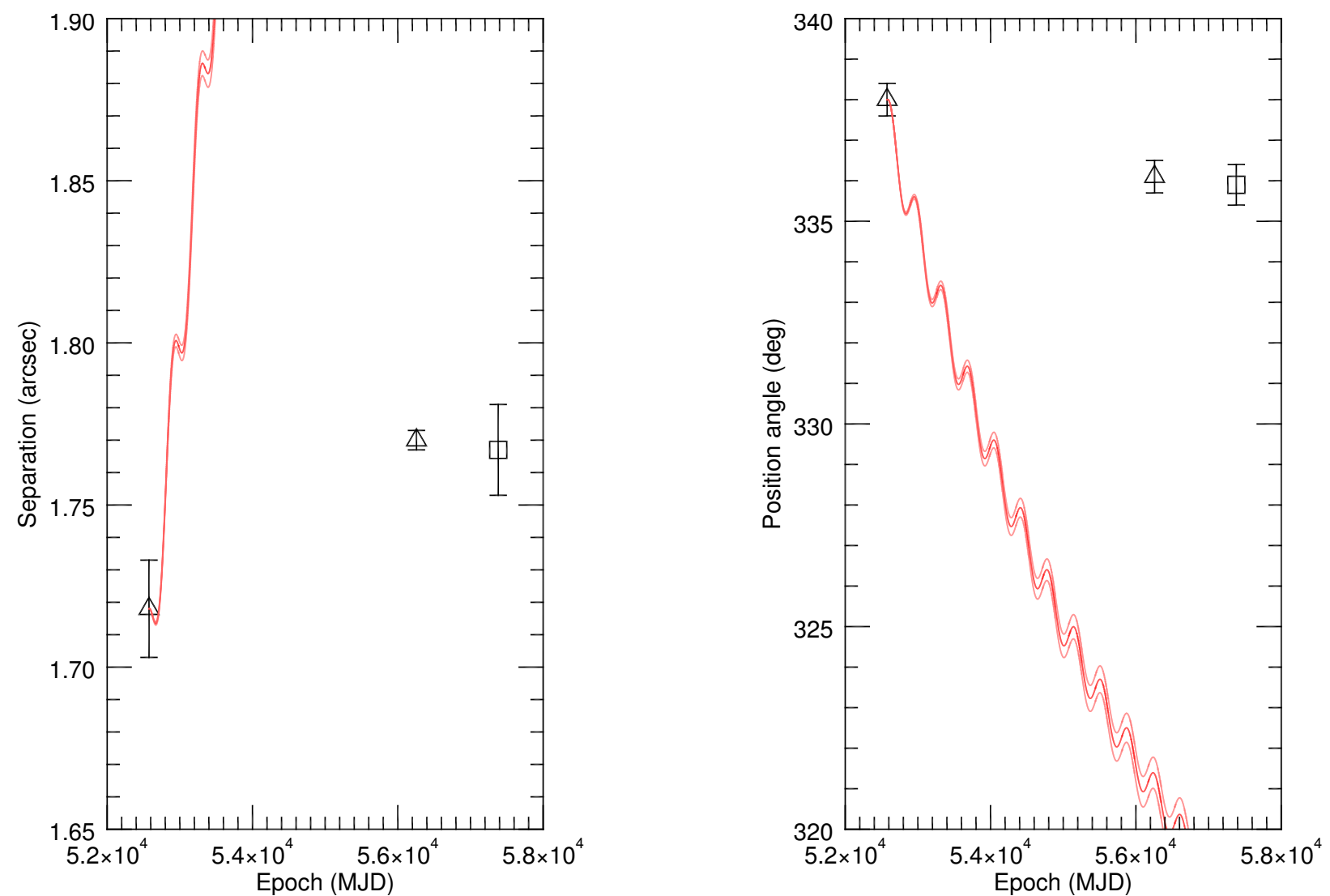

Fig. 3. Separation (left) and position angle (right) of $2 \mathrm{M} 0103(\mathrm{AB}) \mathrm{b}$ relative to the $\mathrm{AB}$ barycenter as functions of time. Triangles are data points from Delorme et al. (2013) and the square in each panel is the astrometry from our AstraLux image. Red lines correspond to the motion that $2 \mathrm{M} 0103(\mathrm{AB}) \mathrm{b}$ would exhibit if it were a static background star, which can be firmly excluded. There is also significant orbital motion between the first epoch in 2002 and the later epochs.

J14142141-1521215: in addition to the three components visible in the AstraLux field of view, there is a wide common proper motion companion at $65^{\prime \prime}$ reported in, e.g., Alonso-Floriano et al. (2015), with identifier 2MASS J14141700-1521125. This wide object happens to be part of the CASTOFFS survey (Schlieder et al. 2012, 2015, and Schlieder et al., in prep.) and thus has FEROS spectroscopy dedicated to it. The spectrum shows signs of high activity, with nearly all of the Balmer series in emission, and very broad lines $(v \sin i=$ $\left.41 \pm 4 \mathrm{~km} \mathrm{~s}^{-1}\right)$. This lends further support to the kinematic indication that the system is young. Exactly how young remains a factor of uncertainty: the classification of J14142141-1521215 in Malo et al. (2013) is for the $\beta$ Pic moving group, but J141417001521125 fits the AB Dor YMG group better.

J15244849-4929473: J15244849-4929473 is single in the AstraLux field of view, but is noted as a single-line spectroscopic binary in Malo et al. (2014). Since no constraints are given on the orbital period, it cannot be assessed whether the unseen companion might become visible to AstraLux during some phase of its orbit.

J17165072-3007104: while this star was identified as a possible ambiguous YMG member in Malo et al. (2013), our re-analysis with BANYAN II implies that it is a young field star with essentially no chance of being part of any of the identified YMGs for any sensible choice of priors. For mass estimations of this resolved binary, we thus arbitrarily chose an age of $150 \mathrm{Myr}$, which is the upper end of the YMG ages used in this study.

J17243644-3152484: this is essentially identical to the case of J17165072-3007104 discussed above: Our BANYAN II reanalysis implies that this star is consistent with a young field star, but it is not possible to associate it directly with any of the known young moving groups. As we did for J17165072-3007104, we use a tentative age of $150 \mathrm{Myr}$.

J18141047-3247344: also known as V4046 Sgr, J181410473247344 is a double-lined spectroscopic binary with a period of 2.4 days (Quast et al. 2000). Furthermore, several studies (Kastner et al. 2011; Alonso-Floriano et al. 2015) have noted that it shares a common proper motion with J181422073246100 , which is individually observed as a separate target in our survey. The separation between the two components is $169^{\prime \prime}$; however, neither is in the detectability range of AstraLux and no other companions are identified.

J19560294-3207186: the two stars J19560438-3207376 and J19560294-3207186, which were separately observed with AstraLux, form a wide binary pair with a separation of $26^{\prime \prime}$. As already noted in Bowler et al. (2015), J19560294-3207186 is itself a close pair, which we confirm in this survey. Furthermore, J19560294-3207186 is noted as a double-lined spectroscopic binary in Elliott et al. (2016), implying that there could be a third component in this close subsystem, and thus a component of a 
quadruple system in total. The system is part of the CASTOFFS survey, in which the spectroscopy in fact shows three distinct lines in absorption and in emission ( $\mathrm{H} \alpha$ and $\mathrm{Ca}$ II $\mathrm{H}$ ) for J19560294-3207186, supporting the quadruple nature of the system.

\section{Summary and conclusions}

In this paper we have presented high-resolution imaging observations of 181 late-type candidate members of nearby YMGs, with the purpose of identifying new binaries that can potentially be used for a range of calibration and age determination purposes. We discovered 61 candidate companions, of which 23 were previously known and the other 38 are new detections. Of the previously known companions, the most notable object is $2 \mathrm{M} 0103(\mathrm{AB}) \mathrm{b}$, which was reported as a $\sim 12-14 M_{\text {jup }}$ wide companion to a close pair of M-dwarfs (Delorme et al. 2013). Our analysis of the $z^{\prime}$ photometry acquired in this work supports the general conclusions in Delorme et al. (2013), but we note that the upper mass limit needs to be extended to $20 M_{\text {jup }}$ when accounting for the full possible age range of the Tuc-Hor association quoted in the recent literature (Torres et al. 2008; Bell et al. 2015). Of all the companions, nine have estimated orbital periods of less than $40 \mathrm{yr}$, and are thus important targets for astrometric follow-ups to constrain their orbital motions.

We also used Gaia data from the recent TGAS release to reanalyze YMG membership for those targets that have new parallactic distances which were not available in the original classifications of the targets. We find that in the majority of cases (17 of 29), the YMG could be confirmed with the new information. Still, in a significant fraction of cases (12 of 29), YMG membership was rejected with the new information. Hence, any individual YMG candidate member without a parallactic distance should be treated with a certain degree of caution. Over the next few years, Gaia will provide distances for all of the targets studied here, thus firmly identifying which systems are bona fide members and which are not. It is also likely to yield more YMG members that had previously been missed, and perhaps new nearby YMGs altogether. Finally, while the closest binaries resolved here will be too close to resolve with the much smaller Gaia, absolute astrometry of the unresolved system can still yield valuable constraints of the orbits when combined with resolved imaging from the ground.

Acknowledgements. M.J. gratefully acknowledges funding from the Knut and Alice Wallenberg Foundation. S.D. acknowledges support from the Northern Ireland Department of Education and Learning. The authors thank the ESO staff for their efficient support, S. Ciceri for transatlantic data transportation, and the anonymous referee for useful suggestions. This study made use of the CDS services SIMBAD and VizieR, the SAO/NASA ADS service, data from the ESA mission Gaia, and digitized archival data from the Anglo-Australian Observatory.

\section{References}

Alonso-Floriano, F. J., Caballero, J., Cortes-Contreras, M., Solano, E., \& Montes, D. 2015, A\&A, 583, A85

Baraffe, I., Chabrier, G., Allard, F., \& Hauschildt, P. H. 1998, A\&A, 337, 403
Baraffe, I., Homeier, D., Allard, F., \& Chabrier, G. 2015, A\&A, 577, A42 Bell, C. P. M., Mamajek, E. E., \& Naylor, T. 2015, MNRAS, 454, 593 Bergfors, C., Brandner, W., Janson, M., et al. 2010, A\&A, 520, A54 Biller, B. A., Liu, M. C., Wahhaj, Z., et al. 2013, ApJ, 777, 160 Bonavita, M., Desidera, S., Thalmann, C., et al. 2016, A\&A, 593, A38 Bonnefoy, M., Chauvin, G., Dumas, C., et al. 2009, A\&A, 506, 799 Bowler, B., Liu, M. C., Shkolnik, E. L., \& Tamura, M. 2015, ApJS, 216, 7 Brandeker, A., Jayawardhana, R., Khavari, P., Haisch, K. E., \& Mardones, D. 2006, ApJ, 652, 1572

Brandt, T. D., Kuzuhara, M., McElwain, M. W., et al. 2014, ApJ, 786, 1

Chauvin, G., Lagrange, A.-M., Bonavita, M., et al. 2010, A\&A, 509, A52 Delfosse, X., Forveille, T., Udry, S., et al. 1999, A\&A, 350, L39 Delorme, P., Gagné, J., Girard, J. H., et al. 2013, A\&A, 553, L5 Elliott, P., Huélamo, N., Bouy, H., et al. 2015, A\&A, 580, A88 Elliott, P., Bayo, A., Melo, C. H. F., et al. 2016, A\&A, 590, A13 Gagné, J., Lafrenière, D., Doyon, R., Malo, L., \& Artigau, É. 2014, ApJ, 783, 121

Gagné, J., Lafrenière, D., Doyon, R., Malo, L., \& Artigau, É. 2015, ApJ, 798, 73

Gaia Collaboration (Brown, A. G. A., et al.) 2016, A\&A, 595, A2

Henry, T. J., Ianna, P. A., Kirkpatrick, J. D., \& Jahreiss, H. 1997, AJ, 114, 388

Hippler, S., Bergfors, C., Brandner, W., et al. 2009, The Messenger, 137, 14 Hormuth, F., Hippler, S., Brandner, W., Wagner, K., \& Henning, T. 2008, SPIE, 7104, 138

Janson, M., Brandner, W., Lenzen, R., et al. 2007, A\&A, 462, 615

Janson, M., Hormuth, F., Bergfors, C., et al. 2012a, ApJ, 754, 44

Janson, M., Carson, J. C., Lafrenière, D., et al. 2012b, ApJ, 747, 116

Janson, M., Bergfors, C., Brandner, W., et al. 2014a, ApJS, 214, 17 Janson, M., Bergfors, C., Brandner, W., et al. 2014b, ApJ, 789, 102 Jayawardhana, R., \& Brandeker, A. 2001, ApJ, 561, L111

Kalas, P., Graham, J. R., Chiang, E., et al. 2008, Science, 322, 1345 Kastner, J. H., Sacco, G. G., Montez, R., et al. 2011, ApJ, 740, L17 Kraus, A. L., \& Ireland, M. 2012, ApJ, 745, 5

Kraus, A. L., Shkolnik, E. L., Allers, K. N., \& Liu, M. C. 2014, AJ, 147, 146 Köhler, R., Ratzka, T., Petr-Gotzens, M. G., \& Correia, S. 2013, A\&A, 558, A80 Kuzuhara, M., Tamura, M., Kudo, M., et al. 2013, ApJ, 774, 11

Lagrange, A.-M., Bonnefoy, M., Chauvin, G., et al. 2010, Science, 329, 57

Law, N. M. 2006, Ph.D. thesis, Institute of Astronomy \& Selwyn College, Cambridge University

Malo, L., Doyon, R., Lafrenière, D., et al. 2013, ApJ, 762, 88

Malo, L., Artigau, E., Doyon, R., et al. 2014, ApJ, 788, 81

Marois, C., Macintosh, B., Barman, T., et al. 2008, Science, 322, 1348

Mason, B. D., Wycoff, G. L., Hartkopf, W. I., Douglass, G. G., \& Worley, C. E. 2001, AJ, 122, 3466

McCaughrean, M. J., \& Stauffer, J. R. 1994, AJ, 108, 1382

Michalik, D., Lindegren, L., \& Hobbs, D. 2015, A\&A, 574, A115

Montet, B. T., Bowler, B. P., Shkolnik, E. L., et al. 2015a, ApJ, 813, L11

Montet, B. T., Morton, T. D., Foreman-Mackey, D., et al. 2015b, ApJ, 809, 25

Perryman, M. A. C., Lindegren, L., Kovalevsky, J., et al. 1997, A\&A, 323, 49

Quast, G. R., Torres, C. A. O., de la Reza, R., et al. 2000, in The Formation of Binary Stars, eds. B. Reipurth, \& H. Zinnecker, IAU Symp., 200, 28

Riaz, B., Gizis, J., \& Harvin, J. 2006, AJ, 132, 866

Sallum, S., Follette, K., Eisner, J. A., et al. 2015, Nature, 527, 342

Schlieder, J. E., Lépine, S., \& Michal, S. 2010, AJ, 140, 119

Schlieder, J. E., Lépine, S., \& Michal, S. 2012, AJ, 143, 80

Schlieder, J. E., Herbst, T. M., Bonnefoy, M., et al. 2015, in 18th Cambridge Workshop on Cool Stars, Stellar Systems, and the Sun, eds. G. van Belle, \& H.C. Harris, 919

Schlieder, J. E., Crossfield, I., Petigura, E., et al. 2016, ApJ, 818, 87

Skrutskie, M. F., Cutri, R. M., Stiening, R., et al. 2006, AJ, 131, 1163

Thalmann, C., Janson, M., Garufi, A., et al. 2016, ApJ, 828, L17

Torres, C. A. O., da Silva, L., Quast, G. R., de la Reza, R., \& Jilinski, E. 2000, AJ, 120,1410

Torres, C. A. O., da Silva, L., Melo, C. H. F., \& Sterzik, M. F. 2008, in Handbook of Star Forming Regions, Vol. II, 757

van der Marel, R. P., Gerssen, J., Guhathakurta, P., Peterson, R. C., \& Gebhardt, K. 2002, AJ, 124, 3255

Zuckerman, B., Song, I., Bessell, M. S., \& Webb, R. A. 2001, ApJ, 562, L87 